\title{
Novel concept of detecting basal cell carcinoma in skin tissue using a continuous-wave millimeter- wave rectangular glass filled probe
}

This article was published in the following Dove Press journal:

Medical Devices: Evidence and Research

\author{
King Yuk Chan \\ Rodica Ramer \\ School of Electrical Engineering and \\ Telecommunications, University of \\ New South Wales, Sydney, NSW, \\ Australia
}

Purpose: This article presents the study and simulation results of a millimeter (mm)-wave device for cancerous tissue detection. mm-Wave approach ensures cheaper equipment instead of the traditional terahertz $(\mathrm{THz})$ frequency approach. A probe that could be implemented using inexpensive silicon technology is proposed, and it also permits integration of entire measuring tool for easy deployment. Skin cancer was chosen as it represents $\sim 80 \%$ of all newly diagnosed cases and is the most common form of cancer in Australia. For an initial development and validation, due to data availability consideration in the open literature, basal cell carcinoma (BCC) was used for simulations.

Methods and results: A probe, using high-frequency signals in the upper mm-wave frequency spectrum (90-300 GHz) to maximize the lateral resolution ( $\mathrm{mm}$ precision) and allows the detection of tumors located at up to $0.5 \mathrm{~mm}$ deep in the skin, is proposed. A frequency-dependent relativity permittivity and an equivalent conductivity of skins were calculated based on the double Debye parameters. For the first time, electromagnetic (EM) models were generated and used along with a high-frequency EM simulator, ANSYS HFSS, to demonstrate the sensitivity of the concept. The following two scenarios were studied: in scenario one, a BCC layer of different thicknesses $(10-3000 \mu \mathrm{m})$ was located on the top of the normal skin and, in scenario two, the BCC was embedded in normal skin at depths from 10 to $3000 \mu \mathrm{m}$. Variability using $\pm 10 \%$ of the corresponding dielectric property was also considered.

Conclusion: This study showed that the reflection coefficients vs frequency could capture useful information indicating the possible presence of BCC at mm-wave frequencies. Both magnitude and phase of the reflection coefficient were quantified, with two scenarios analyzed. It was found that a dual-band approach, $100-150$ and $200-250 \mathrm{GHz}$, has the ability to highlight deviations from the normal skin.

Keywords: millimeter wave, sensor, electromagnetics, near-field, continuous wave, diagnostic

\section{Introduction}

Skin cancer is the most common form of cancer in Australia, representing $\sim 80 \%$ of all newly diagnosed cases, ${ }^{1}$ with melanoma accounted for $>10 \%$ of all reported incidence, in $2014^{2}$ and $2017 .{ }^{3}$ The majority of skin cancers are detected by patients themselves, or by their partners, while some are detected by physicians during the routine clinical examinations. There is no doubt that early detection could significantly reduce complications or mortality.

There are many patients in Australia who are known to be at moderate-to-high risk of nonmelanoma skin cancer or who have pigmented lesions, which are at risk of developing into melanoma. Today, it is possible to use dermoscopy to determine if the
Correspondence: King Yuk Chan School of Electrical Engineering and New South Wales, Sydney, 2052 NSW, Australia

Tel +6l $2938509 \mid 4$

Email kyc@unsw.edu.au 
lesions are benign or suspicious and if they require excision for biopsy for conclusive diagnosis. However, it is more often the case that patients have lesions, which do not fulfill the criteria of malignancy but need ongoing monitoring. As with objective blood tests, which are reliable means of monitoring patients at risk of complications (such as deteriorating kidney function), irrespective of the circumstances of their medical examination, ideally, there should exist an accurate mean of assessing the patients for skin cancer too. Under the present health care system, no such objective measure exists.

Prospective total skin photography has been advocated as one method of picking up the onset of melanoma in patients with many atypical moles since it is more often the case that melanoma arises as a new lesion rather than as a changed existing lesion. However, when one has hundreds of moles, many of which are located on the back, it is unlikely that either the patient or the doctor who sees the patient once every 6 months can investigate all lesions without expensive photography and detailed review. If supplementary, detailed dermoscopic images are taken to investigate certain moles, specialized equipment and software are required at extra cost.

Existing imaging techniques for skin cancer diagnosis (such as total body photography and sequential imaging at the macroscopic level) have a low resolution. ${ }^{4,5}$ At the microscopic level, where higher resolution imaging can be achieved, invasive biopsy samples need to be imaged to determine the type and stage of the skin cancer.

The everlasting desire to improve the quality of life has heightened the competition to develop imaging techniques for health monitoring. Currently, optical coherence tomography, ultrasound, near-IR, and Raman spectroscopies, MRI, positron emission tomography, and X-ray techniques are receiving the most attention. ${ }^{6-14}$ Some of these techniques offer nonionizing solutions, good resolution, good acquisition speeds, and well-targeted contrast mechanisms. However, none of them meets all of these crucial criteria simultaneously. Besides, most of them require costly and bulky equipment and tools.

Hence, it would be advantageous if there were a device, which could be used with a validated read out score, to indicate if particular lesions have reached the "at-risk" stage. This is of particular importance because, in Australia, there is an extensive list of patients waiting to be seen by a dermatologist. As a result, a large number of lesions are unnecessarily removed because of medicolegal and unstated financial incentives.

To overcome the problems above, we propose here the design of a practical small handheld device able to detect the responses of skin conditions (normal and basal cell carcinoma $[\mathrm{BCC}])$ from electromagnetic $(\mathrm{EM})$ radiation at high mm-wave to low terahertz $(\mathrm{THz})$ frequencies. This design represents a preamble for a follow-up sensitive, an integrated readout device, that can be made portable and that allows its deployment everywhere and anytime by any medical staff. A silicon millimeter ( $\mathrm{mm}$ ) mm-wave integrated circuit-type-based technology will ensure the reduced cost for mass deployment and implementation. This first study was conducted heavily relying on the existing open available data on BCC EM parameters. It is hoped that its success will give sufficient experience and background for other lethal cancers' electronic devices' studies.

\section{Background}

The high mm-wave to low $\mathrm{THz}$ signal range was chosen for our studies as it provides some unique features that make it particularly suitable for medical applications. For example, mm-waves have low generated photon energy ${ }^{15,16}$ not causing chemical damage to molecules or harmful ionization in biological tissues. Studies of high-power EM waves on human mammalian cells observed no significant differences between exposed and nonexposed cells, other than small temperature changes. ${ }^{17}$ Also, mm-waves have very high absorption rates in water, marking them as responsive to characterize polar substances such as water and hydrated matter. ${ }^{18,19}$ Mm-waves' manipulation entails also smaller device sizes that are beneficial for cheap mass production.

We propose a novel device that would permit designing of a system that could quantify the reflected signal from the exposed skin area. The proposed technique is based on shining the skin tissue to a mm-wave so that the complex dielectric properties can be evaluated from this interaction. For different real and imaginary values of different skins, different reflections and absorptions occur, providing unique signatures. McAuley et al and others ${ }^{20-22}$ showed that mmwave is able to provide high contrast on tissue depending on their water content. This contrast has been exploited before to map the water distribution in plants ${ }^{23}$ and severely burnt skin samples. ${ }^{21}$ Medical researchers have found that certain tumors have higher water content compared to healthy tissues. ${ }^{24-27}$ Researchers also showed that the water absorption of the mm-wave signal increases significantly with temperature. ${ }^{28}$ Also, it has been concluded that in vivo tissue generally has higher water content compared to ex vivo tissue. ${ }^{29}$ Therefore, the in vivo losses are more significant than the equivalent ones in the ex vivo samples at the room temperature.

During the past 2 decades, groups from around the globe conducted research on imaging with mm-waves and $\mathrm{THz}$ 
waves. Most of their approaches used $\mathrm{THz}$ pulsed imaging (TPI) with optical mixing to generate higher mm-wave and $\mathrm{THz}$ signals and performed the analysis with finite difference time domain method. ${ }^{20,30-32}$ Currently, there is only one commercially available tissue imager, of great significance in the world, produced by TeraView. ${ }^{33}$ The main drawbacks of using optical mixing systems are that they are bulky, expensive, and immovable. This is because these systems are primarily based on optical sources, mirrors, and lenses. To overcome these problems, more recent research shifts the focus to nearfield systems that use mm-waves' circuits. Sensors operating $<40 \mathrm{GHz}$ have been proposed and demonstrated; although they provide well-integrated solutions, their sensitivity is insufficient, due to the relatively large wavelengths. ${ }^{34-36}$ Other systems, operating at much higher frequencies $\sim 90 \mathrm{GHz}$, have shown promising results ${ }^{22,37}$ but are very bulky and not suitable for integration in a readout device with the small form factor.

In recent years, mm-wave technology has undergone dramatic advancements mostly as a result of the progress in silicon transistors and integrated circuits, making them suitable for operation throughout the entire mm-wave spectrum (30-300 GHz). Several large volume silicon mm-wave products, such as automotive cruise control radars ${ }^{38,39}$ and $60 \mathrm{GHz}$ wireless USB3 transceivers operating at $5 \mathrm{~Gb} / \mathrm{s}$ rates, ${ }^{40}$ have demonstrated the feasibility and resilience of mm-wave silicon technology in the market. The costs of mm-wave silicon transceivers have also significantly diminished. Silicon mm-wave integrated circuits are now poised to take on and revolutionize a variety of new applications, where the medical imaging and diagnosis are among the most promising.

\section{Methods}

The suggested system consists of the following three parts: a signal generator-detector (eg, vector network analyser [VNA]), the proposed device itself, called "the probe", and the sample under test (skin area). The general practitioners or qualified personnel can use the probe by scanning it over the visually examined area of investigation. The key innovation of this work consists in designing a probe that offers a small footprint for high lateral resolution sensing with sufficient sensitivity for high contrast between the normal and diseased skin and also in the EM modeling of the $\mathrm{BCC}$ using existing data from the literature. The system is designed based on the principle of continuous-wave (CW) near-field EM wave reflectometry. The generated mm-wave signal illuminates the skin sample and travels through the probe; a part of the signal penetrates the sample with the remainder reflected through the probe and reached back the detector. This reflected signal gives unique information (in terms of its amplitude and phase) at different frequencies when compared with the transmitted signal from the generator. The deviances of the magnitude and phase of different skin samples from the normal skin are determined in this article. In the suggested system, the signal generator-detector could be a commercially available VNA, with the final aim to fabricate a fully integrated circuit combining the VNA function with the probe scanning feature, as future work. The probe is designed so that it permits limited penetration depth within typical skin thickness (eg, <3 mm). ${ }^{41,42,43}$ The normal dry skin and the most common type of skin cancers BCC were considered in this study. For simplicity, a flat and homogeneous skin was assumed. The assumptions are valid provided that the simulated skin area $(1.5 \mathrm{~mm}$ in diameter) is much smaller than the typical average BCC size, which is $\sim 3-5 \mathrm{~mm}$ in diameter. ${ }^{41}$ Also, the total skin thickness that consists of normal and BCC layers was limited here to $3 \mathrm{~mm}$, as this is the typical maximum skin (epidermis-dermis) thickness across different body parts. ${ }^{41,42}$

There have been three steps in the present study. First, we calculated the variation of relative electrical permittivity and total effective conductivity functions vs frequencies. Second, the near-field reflectometry probe was designed. Finally, the combined probe-skin samples (treated as terminated/loaded probes) were studied and followed by their high frequency structure simulator (HFSS) simulations. The results accomplished in these steps are outlined later.

\section{Step one: normal skin and BCC electrical parameters calculation}

With CW EM simulations, typical commercially available software packages (eg, HFSS and CST) are unable to use complex frequency-dependent electric permittivity functions. Instead, they require the relative permittivity $\varepsilon_{\mathrm{r}}$ and the electric conductivity $\sigma$.

However, human skin dielectric properties are typically modeled as complex frequency-dependent permittivity $\varepsilon^{*}(\omega)$, based on Debye theory. Pickwell et a ${ }^{44}$ used double Debye theory and modeled frequency-dependent permittivity $\varepsilon^{*}(\omega)$, from 0.1 to $4 \mathrm{THz}$ for both normal skin and $\mathrm{BCC}$, where $\varepsilon^{*}(\omega)$ is given by

$$
\varepsilon^{*}(\omega)=\varepsilon_{\infty}+\frac{\varepsilon_{\mathrm{s}}-\varepsilon_{2}}{1+j \omega \tau_{1}}+\frac{\varepsilon_{2}-\varepsilon_{\infty}}{1+j \omega \tau_{2}}
$$

where $\varepsilon_{\mathrm{s}}, \varepsilon_{\infty}, \varepsilon_{2}, \tau_{1}$, and $\tau_{2}$ are the five double Debye parameters.

Table 1 shows the double Debye parameters that Pickwell obtained for in vivo healthy and BCC skin samples. Pickwell 
was able to use these parameters to perform simulations in the time domain with optical techniques to verify their designs that ultimately resulted in producing the only commercially available clinical hardware for skin cancer detection using TPI. ${ }^{33}$

In our study, we perform EM modeling using Pickwell's double Debye parameters. Equation 1 was separated into the real $\left(\varepsilon^{\prime}\right)$ and imaginary $\left(\varepsilon^{\prime \prime}\right)$ parts as in Equation (2):

$$
\varepsilon^{*}=\varepsilon^{\prime}-j \varepsilon^{\prime \prime}
$$

The real part $\varepsilon^{\prime}$ represents the relative permittivity or $\varepsilon_{\mathrm{r}}$, and the imaginary part $\varepsilon^{\prime \prime}$ was further expressed as total effective conductivity (equivalent conductivity); permittivity is the ability of a substance to store electrical energy in an electric field. Electrical permittivity of free space is a universal constant $\varepsilon_{0}=8.854 \times 10^{-12} \mathrm{~F} / \mathrm{m}$. Materials are characterized by the electrical permittivity $\varepsilon_{\mathrm{r}} \varepsilon_{0}=\varepsilon^{\prime}[\mathrm{F} / \mathrm{m}]$ and $\varepsilon_{\mathrm{r}}$ is the relative permittivity and is $>1$. Materials can also be characterized by losses, which include dielectric damping and conductive loss. Together they form $\varepsilon^{\prime \prime}$ and that can be expressed as the total effective conductivity equivalent conductivity. $\sigma_{\mathrm{e}}$ of the material (skin) as in Equation (3)

$$
\varepsilon^{\prime \prime}=\frac{\sigma_{\mathrm{e}}}{2 \pi f \varepsilon_{0}}
$$

where $\varepsilon_{0}=8.85 \times 10^{-12} \mathrm{~F} / \mathrm{m}$ is the permittivity of free space and $f$ is the frequency in $\mathrm{Hz}$.

It should be noted that this total effective conductivity is not just the static (ionic) conductivity; it includes all the dielectric losses such as the standard static (ionic) conductivity and the associated loss (imaginary) terms from double Debye theory.

These calculations have been performed for each frequency point, ranging from 95 to $300 \mathrm{GHz}$, and are plotted in Figure 1. In order to account for variability, $\pm 10 \%$ of the BCC's relative permittivity $\varepsilon_{\mathrm{r}}$ and the total effective conductivity $\sigma_{\mathrm{e}}$ were also included.

\section{Step two: probe considerations}

A typical coaxial type probe could be used as the sensor. Although some coaxial probes are commercially available, they are not suitable for this application as they suffer from

A

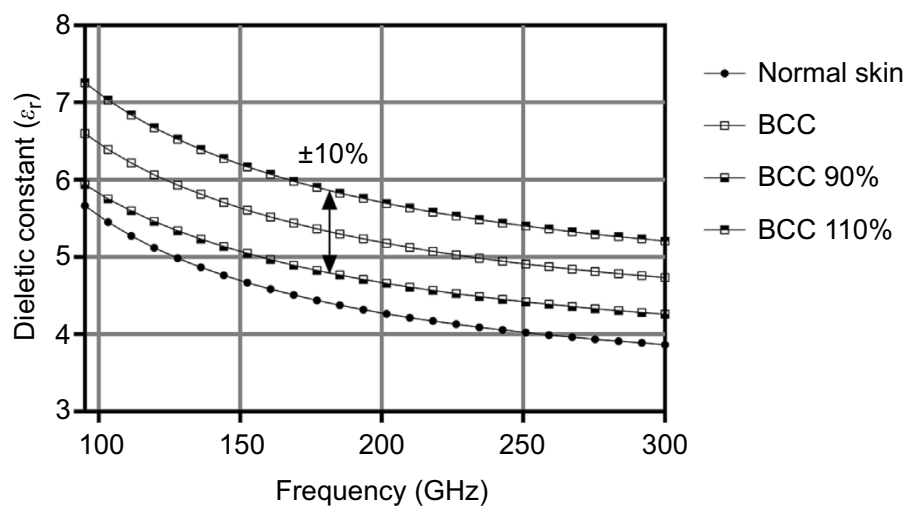

B

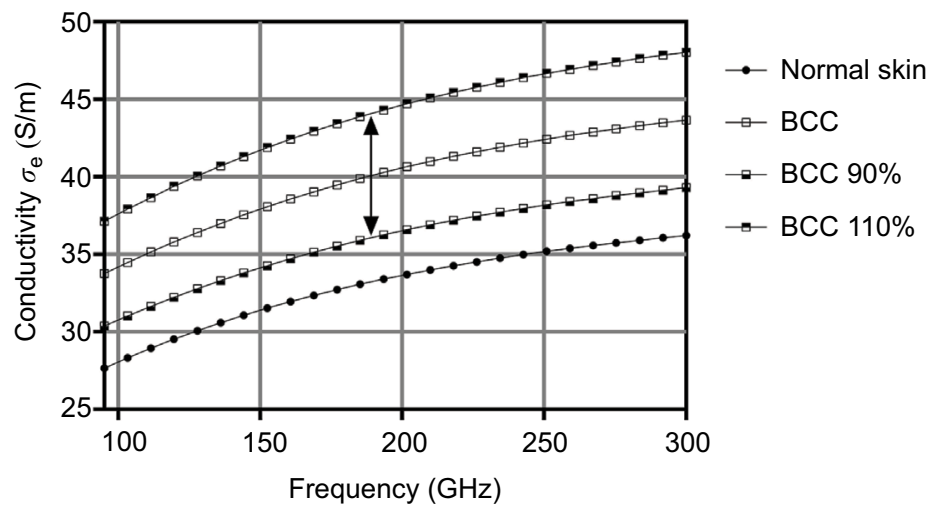

Figure I (A) Extracted frequency-dependent relative permittivity $\varepsilon_{\mathrm{r}}$ and (B) total effective conductivity (equivalent conductivity) $\sigma_{\mathrm{e}}(\mathrm{S} / \mathrm{m}$ ) for normal dry skin and BCC using the double Debye parameters by Pickwell for frequencies ranging from 95 to $300 \mathrm{GHz}$.

Abbreviation: $\mathrm{BCC}$, basal cell carcinoma. 
Table I Double Debye parameters for normal skin and BCC for the calculation of the frequency-dependent relative electric permittivity $\varepsilon_{\mathrm{r}}$ and total effective conductivity (equivalent conductivity) $\sigma_{\mathrm{e}}$

\begin{tabular}{llllll}
\hline Material & $\varepsilon_{\alpha}$ & $\varepsilon_{\mathrm{s}}$ & $\varepsilon_{2}$ & $\tau_{1}(\mathbf{p s})$ & $\tau_{2}(\mathbf{p s})$ \\
\hline Normal skin & 3.4 & 25 & 5 & 7 & $\mathrm{I}$ \\
BCC & 4.2 & 40 & 6.2 & 10 & $\mathrm{I}$ \\
\hline
\end{tabular}

Abbreviation: $\mathrm{BCC}$, basal cell carcinoma.
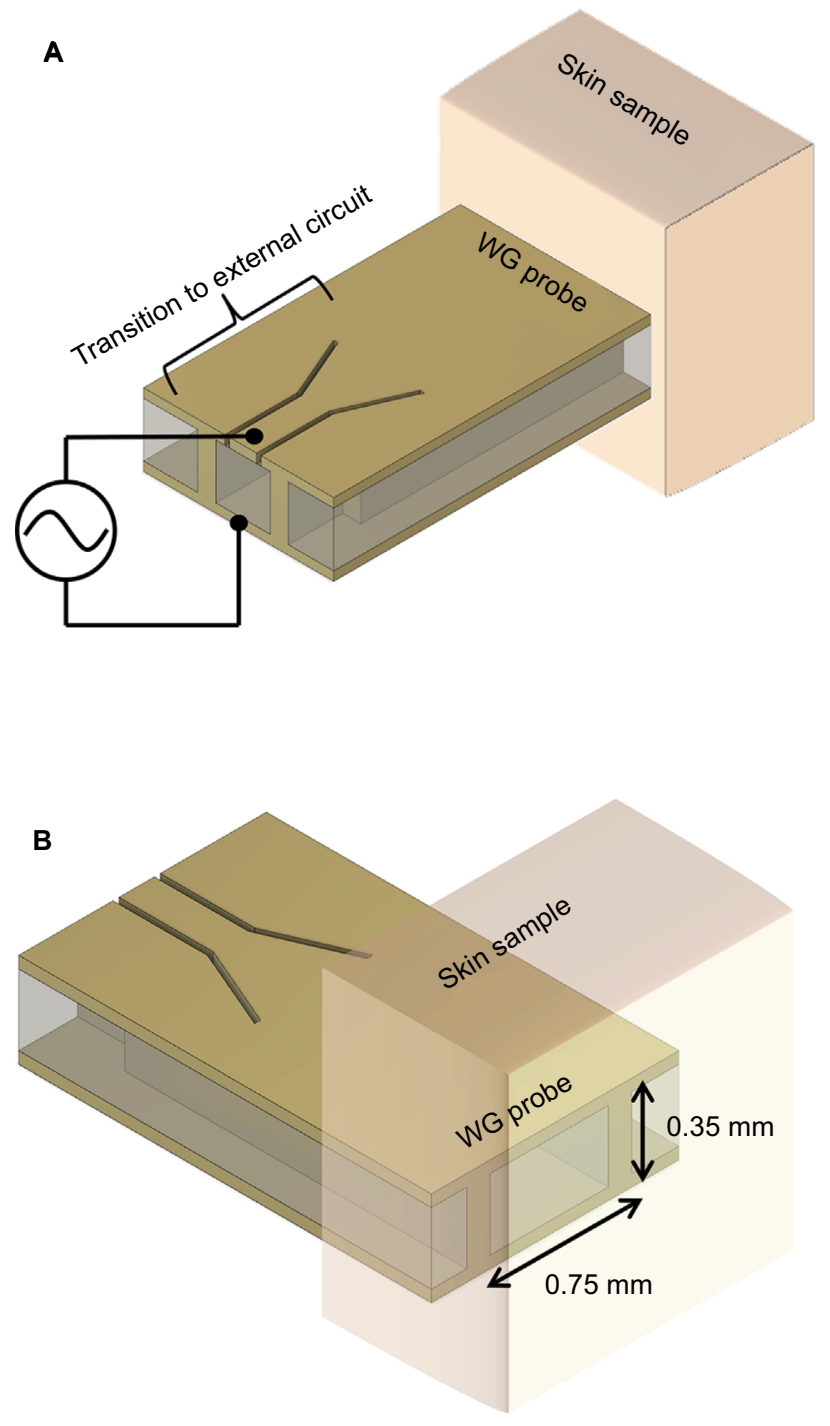

Figure 2 Probe sitting on the skin sample of interest.

Notes: (A) Front view of the probe connected to the VNA via a CPW transition. (B) Back view showing the rectangular waveguide behind the skin sample.

Abbreviations: CPW, coplanar waveguide; VNA, vector network analyser; WG, waveguide.

having relatively large footprints. ${ }^{45}$ Typically $>3 \mathrm{~mm}$ in diameter, the commercial probes, which will also sense surrounding normal skin due to the fringing field, are not able to provide sufficient discrimination between normal skin, the BCC properties, and the surrounding normal skin. ${ }^{46}$ Figure
2 illustrates the proposed rectangular waveguide probe that supports the transverse electric $\mathrm{TE}_{10}$ mode for the sensing signal and that is easy to fabricate. The rectangular waveguide probe sits on the skin area of interest at one end and is connected to the VNA (generator and detector) through a coplanar waveguide transition at the other end. Front and back views of the skin sample in contact with the probe are shown in Figure 2A and B.

A higher operational frequency, $>90 \mathrm{GHz}$, is selected based on the lateral resolution and penetration considerations. In rectangular waveguide design, higher frequencies offer smaller waveguide cross-section due to waveguide cutoff frequency ${ }^{44}$ and also provide shallower EM wave penetration in the skin. The lateral resolution and EM penetration must be adequately tackled. This is because BCC can be very shallow with a thickness in sub-mm range and can have a lateral dimensions of order of $\mathrm{mm}^{2}$. Glass was selected as the waveguide filling material due to its wide commercial availability for microfabrication. Glass also has a dielectric constant of 5.5, which is the closest to 5.6 value that is attributed to the normal skin, ${ }^{47}$ allowing the minimization of the impedance mismatch between the probe and normal skin at the contact interface. This permits the mm-wave signal to penetrate into the skin and allows to take place only when mismatch ${ }^{46}$ occurs. As BCC exhibits departure from the dielectric constant and conductivity values of the normal skin, a mismatch will be encountered and this can be quantified by measuring the reflection coefficient.

With the selection of sensing frequencies $>95 \mathrm{GHz}$ and glass as filling material for the probe, a minimal footprint, as small as $0.7 \mathrm{~mm}(W) \times 0.35 \mathrm{~mm}(H)$ can be achieved. Also, as the sensing frequency is $>95 \mathrm{GHz}$ and operation is in the near-field EM radiation condition, the system is interference immune. It will offer interference avoidance from existing far-field communication and military systems, operating at frequencies $<100 \mathrm{GHz}$.

\section{Step three: probe loaded with skin samples study}

An entire combination of the probe loaded with different skin samples was simulated in HFSS. The EM signal was excited from the generator (the VNA), has traveled through the probe, was reflected from the skin, and has returned back to the detector (the VNA). Two possible occurring scenarios were considered in order to emulate different BCC conditions.

In scenario 1, a shallow BCC layer of various thicknesses is located on the top of the normal skin, as shown in Figure 3A. Figure $3 \mathrm{~B}$ and $\mathrm{C}$ shows the EM simulation set-up and the $\mathrm{TE}_{10}$ mode propagation for scenario 1 . 
A

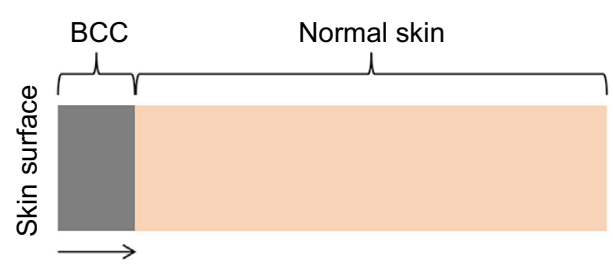

Varying BCC thickness
B

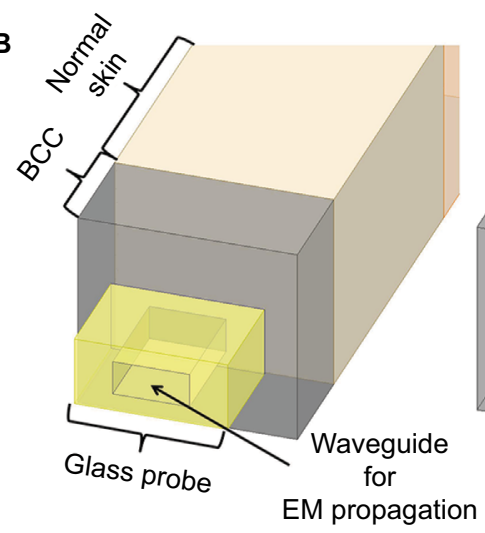

E

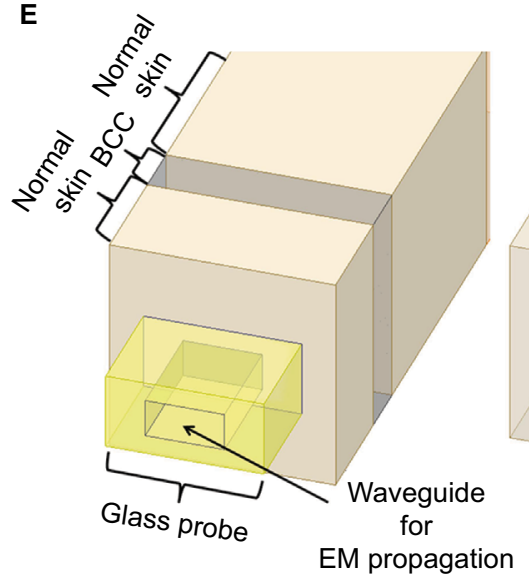

EM propagation
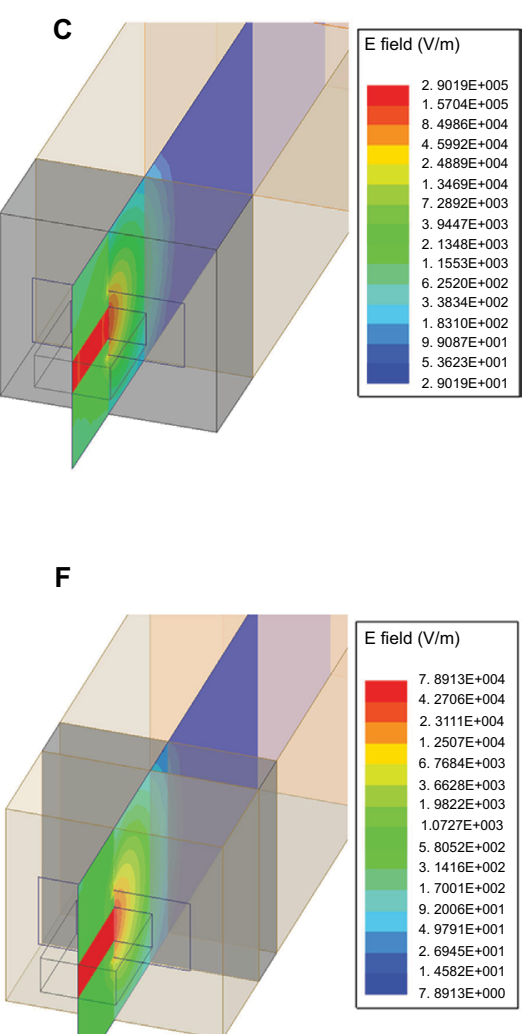

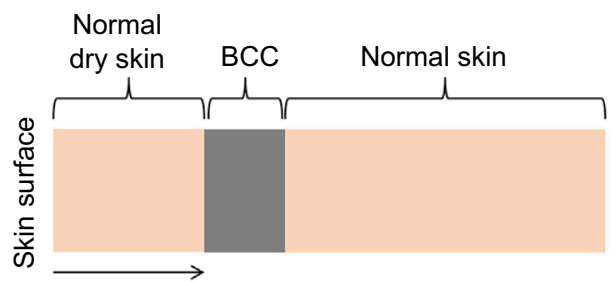

Varying depth of BCC layer

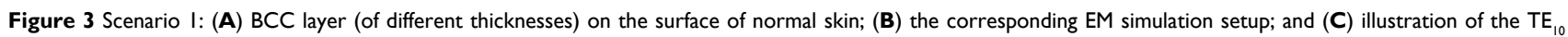

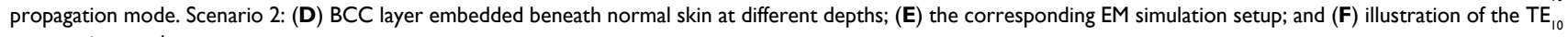
propagation mode.

Abbreviations: BCC, basal cell carcinoma; EM, electromagnetic.

In scenario 2, a $500 \mu \mathrm{m}$ thick BCC layer is embedded under the normal skin at various depths, as shown in Figure 3D. Figure $3 \mathrm{E}$ and $\mathrm{F}$ shows the EM simulation set-up and the illustration of the $\mathrm{TE}_{10}$ mode propagation for scenario 2 .

\section{Results and discussion}

The HFSS-simulated reflected signals were analyzed in terms of the magnitude $\left(\left|S_{11}\right|\right.$ in $\left.\mathrm{dB}\right)$ and phase $\left(\angle S_{11}\right.$ in degrees) of the reflection coefficient, for both scenarios 1 and 2 . These results were compared with the magnitude $\left|S_{11}\right|$ and phase $\angle S_{11}$ of the reflection coefficient for the normal skin that are given in Figure 4.

We note that for frequency ranging between 150 and $300 \mathrm{GHz}$, the magnitude $\left|S_{11}\right|$ takes values $<-20 \mathrm{~dB}$. Therefore, in order to effectively emphasize the effectiveness of the proposed system, the results were presented as differences between the magnitudes and phases of the reflection coef- ficients where the $\mathrm{BCC}$ values were considered first and then the normal skin, ie, $\Delta\left|S_{11}\right|=\left|S_{11}\right|_{\mathrm{BCC}}-\left|S_{11}\right|_{\text {normal skin }}$ and $\Delta \angle S_{11}=$ $\angle S_{11 \mathrm{BCC}}-\angle S_{11 \text { normal skin }}$. The study results for scenarios 1 and 2 are illustrated in Figures 5 and 6 , where $\pm 10 \%$ variations in relative permittivity $\varepsilon_{\mathrm{r}}$ and total effective conductivity $\sigma_{\mathrm{e}}$ were introduced (eg, $\varepsilon_{\mathrm{r}}=90 \%$ and $110 \%$ and $\sigma_{\mathrm{e}}=90 \%$ and $110 \%$ of the original calculated functions), to account for possible variability.

For scenario 1, Figure 5A-E shows the differences in magnitude of the reflection coefficient $\Delta\left|S_{11}\right|$, while Figure 5F-J illustrates the phase differences $\Delta \angle S_{11}$ of BCC of different thicknesses $(10,50,100,500,1000$, and $3000 \mu \mathrm{m})$ and normal skin, over the entire frequency range, with $\pm 10 \%$ variations in relative permittivity $\varepsilon_{\mathrm{r}}$ and conductivity $\sigma_{\mathrm{e}}$ of the BCC. According to these results, even a very shallow BCC layer resulted in a distinguishable difference in magnitude response compared to normal skin. This is 


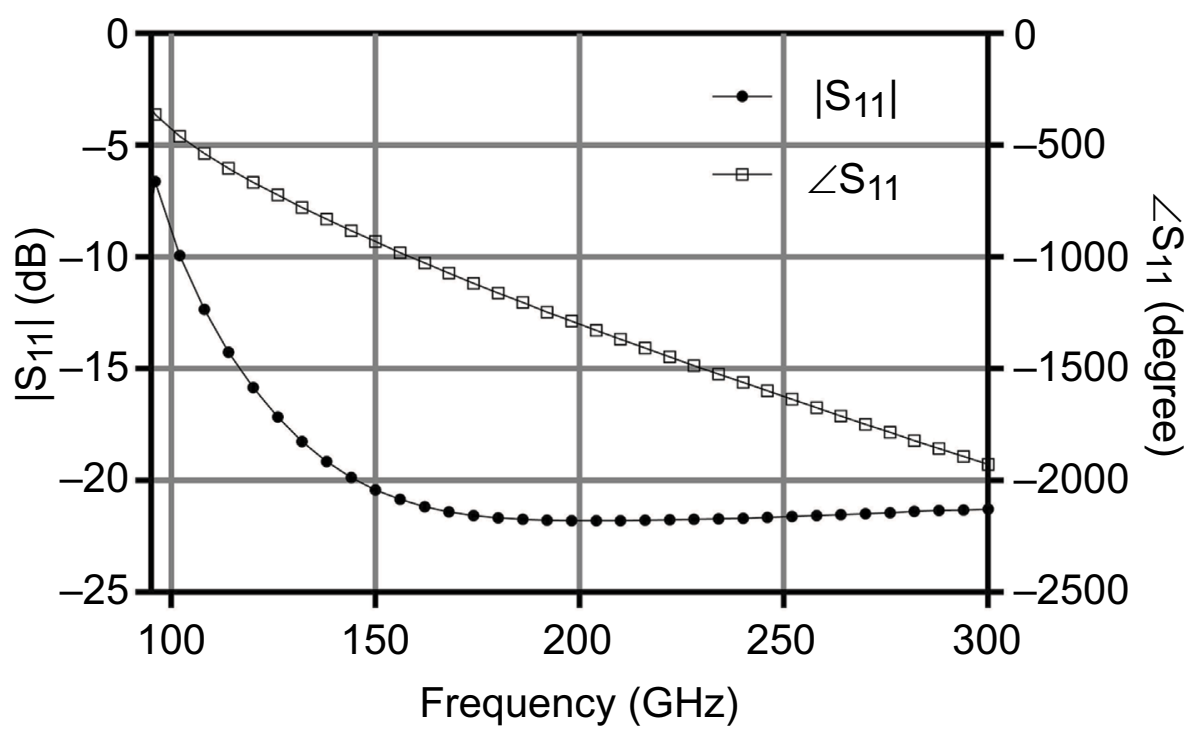

Figure 4 HFSS simulation results: magnitude $\left|S_{1 \mid}\right|(d B)$ and phase $\angle S_{11}$ (degree) of the reflection coefficient for probe loaded with the normal skin.

even more pronounced for the difference in phase response, where other than the $10 \mu \mathrm{m}$ thin BCC layer, a large phase deviation from the normal skin phase is observed, in particular $>200 \mathrm{GHz}$.

Figure $6 \mathrm{~A}-\mathrm{J}$ shows the simulation results for the second scenario, where a $500 \mu \mathrm{m}$ thick BCC layer is embedded in a normal skin layer at progressive depths 10, 50, 100, 500,1000 , and $3000 \mu \mathrm{m}$ with $\pm 10 \%$ variations in relative permittivity $\varepsilon_{\mathrm{r}}$ and conductivity $\sigma_{\mathrm{e}}$ of the BCC. Clearly, notable differences in magnitude $\left|S_{11}\right|$ (Figure 6A-E) and phase $\angle S_{11}$ (Figure $6 \mathrm{~F}-\mathrm{J}$ ) values could be captured for all BCC layers embedded at depths from 10 to $100 \mu \mathrm{m}$, within these variations. In fact, the shallower the BCC layer is embedded under normal skin tissue, the more differences to the $\Delta\left|S_{11}\right|$ and $\Delta \angle S_{11}$ from those for the normal skin. It should be noted that according to the simulations, as the BCC layer is embedded $>500 \mu \mathrm{m}$ under the normal skin, the responses from both magnitude and phase become similar to normal skin, across the entire $95-300 \mathrm{GHz}$ frequency range. This is, in fact, a desirable feature for the proposed probe. According to researchers, ${ }^{48}$ the device should only detect unusual tissues that are embedded in the epidermis layer, which has a typical thickness of $<100 \mu \mathrm{m}$ across body sites.

The difference in magnitude shows $<2 \mathrm{~dB}$ values $>200 \mathrm{GHz}$ for scenario 1 , and the difference in magnitude shows $>3 \mathrm{~dB}$ values $<200 \mathrm{GHz}$ for scenario 2 , as shown in Figures 5 and 6, respectively. As magnitude $\left|S_{11}\right|$ for the reference normal skin is $<-20 \mathrm{~dB}>200 \mathrm{GHz}$, the absolute magnitudes for both scenarios are only $\sim-20 \mathrm{~dB}$, which correspond to very small values. This is not the case for the differences in phase, $\Delta \angle S_{11}$ data, where significant values were obtained, rendering the phase as the preferred parameter for our analysis. Therefore, a dual-band operation (95-150 and 200-250 GHz) is ideal, where magnitude and phase are the preferred parameters, respectively, for the BCC detection. Dual-band approach will not pose any difficulty in silicon technology implementation. ${ }^{49}$

With the proposed concept, the following advantages could be achieved: The small footprint of the probe for high lateral resolution $(\sim \mathrm{mm})$ sensing; Controlled sensing depth into the skin, suitable for $100 \mu \mathrm{m}$ deep embedded BCC; High contrast between normal skin and BCC, for satisfactory sensitivity; Cheap silicon technology implementation; Integration with on-chip VNA for an ultimate small, hand-held portable device that can easily be deployed anywhere.

The present study reveals that the proposed technique has the potential to detect BCC presence; however, at this stage, it cannot provide absolute location and thickness information. In fact, as suggested in Truong et al, ${ }^{50}$ normal skin and BCC could result in very different dielectric properties depending on the person, the body parts, modeling, and measurement techniques. Therefore, this proposed technique is valid and 
A
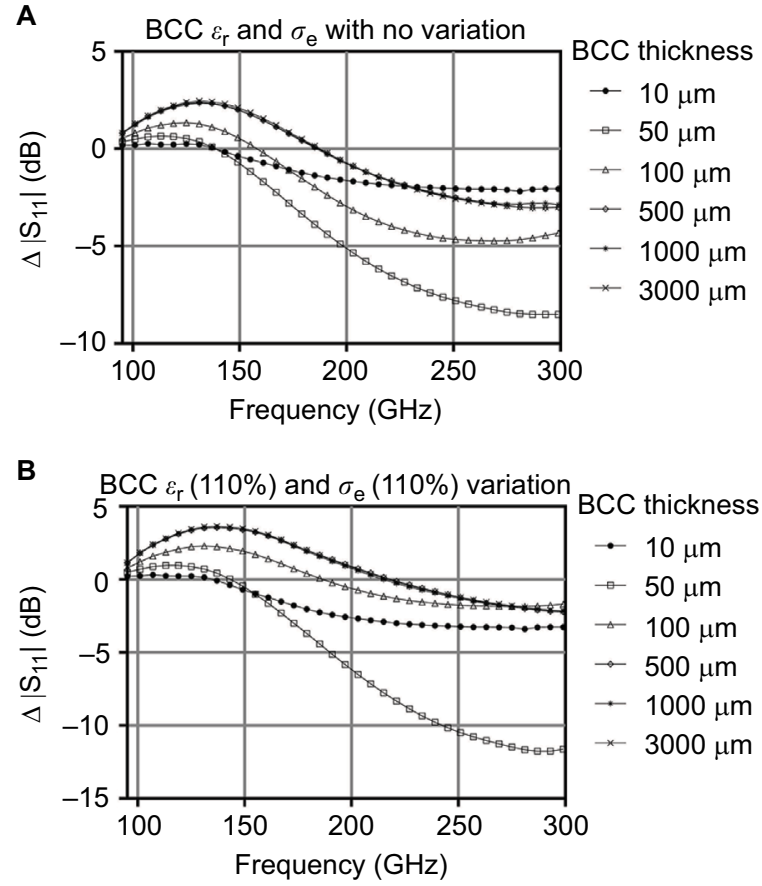

C

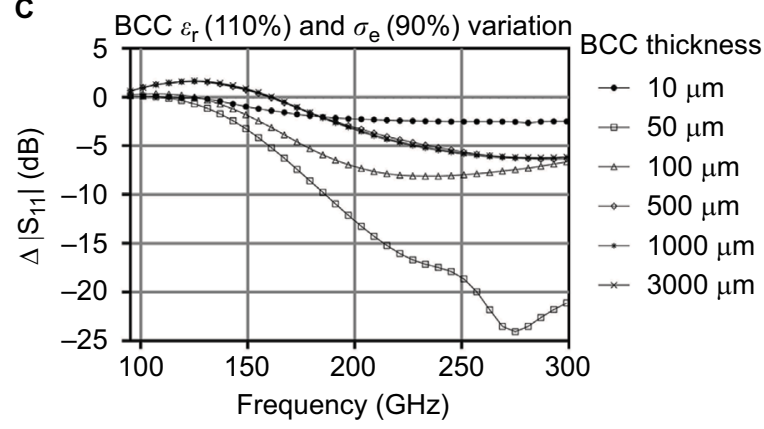

D

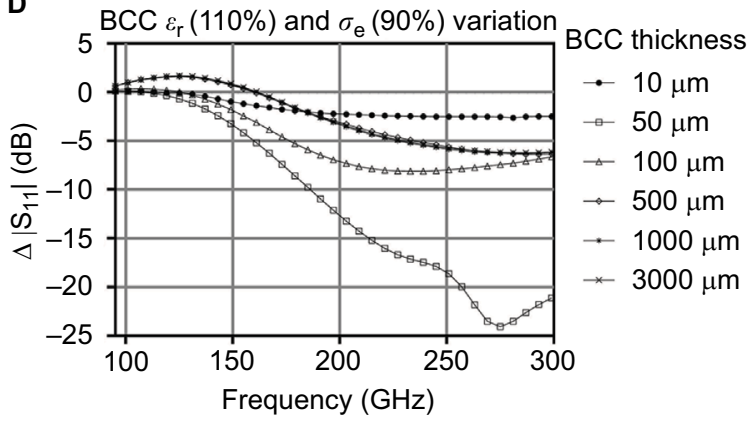

E

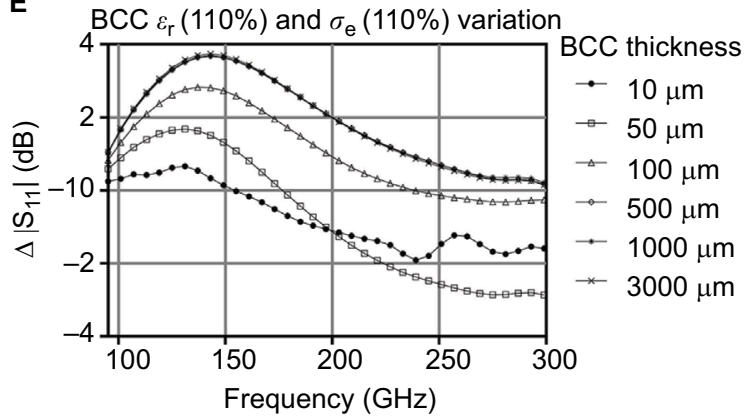

F

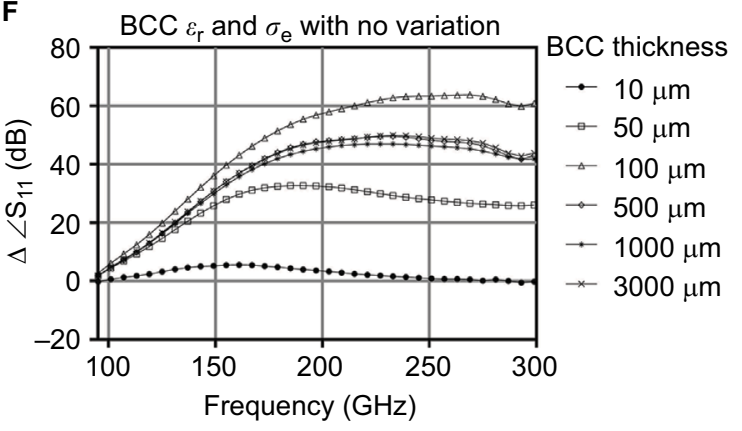

G $\mathrm{BCC} \varepsilon_{\mathrm{r}}(110 \%)$ and $\sigma_{\mathrm{e}}(110 \%)$ variation $\mathrm{BCC}$ thickness

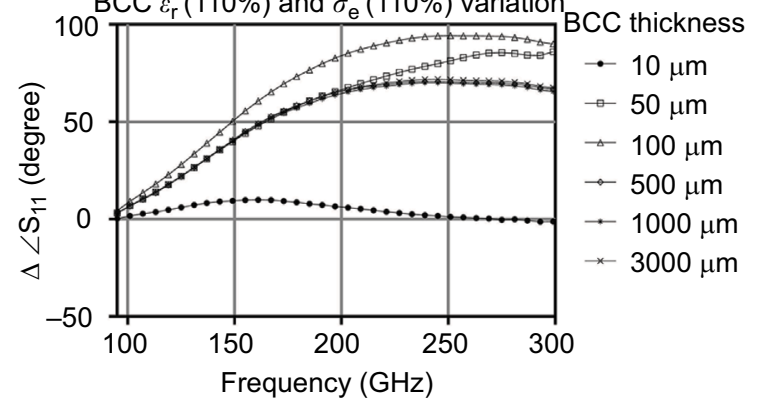

H $\mathrm{BCC} \varepsilon_{\mathrm{r}}(110 \%)$ and $\sigma_{\mathrm{e}}(90 \%)$ variation $\mathrm{BCC}$ thickness

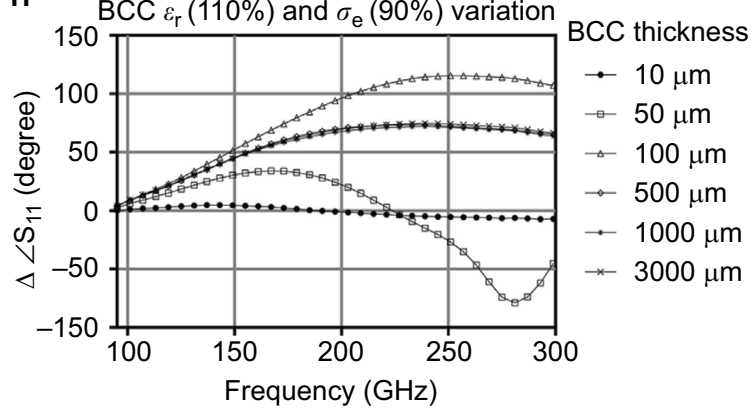

I $\mathrm{BCC} \varepsilon_{\mathrm{r}}(90 \%)$ and $\sigma_{\mathrm{e}}(90 \%)$ variation $\mathrm{BCC}$ thickness

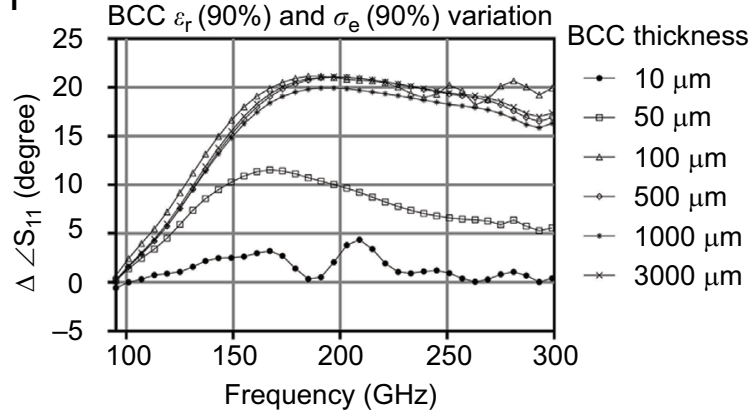

J

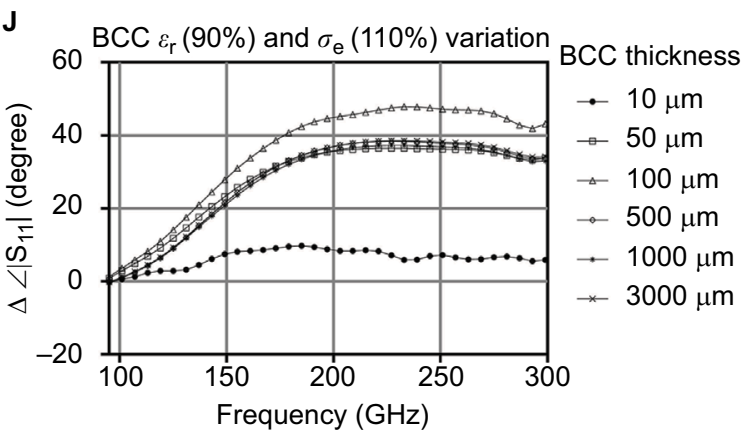

Figure 5 Simulation results of scenario I (BCC layer with thickness 10, 50, 100, 500, 1000, and $3000 \mu \mathrm{m}$ on the surface of healthy skin): (A-E) shows differences in magnitude $(\Delta|\mathrm{SI} I|)$ and $(\mathbf{F}-\mathrm{J})$ shows phase $(\Delta \angle \mathrm{SI} \mathrm{I})$ of the reflection coefficient, with $\pm \mathrm{I} 0 \%$ variation of $\mathrm{BCC}$ 's $\varepsilon_{\mathrm{r}}$ and $\sigma_{\mathrm{e}}$.

Abbreviation: BCC, basal cell carcinoma. 

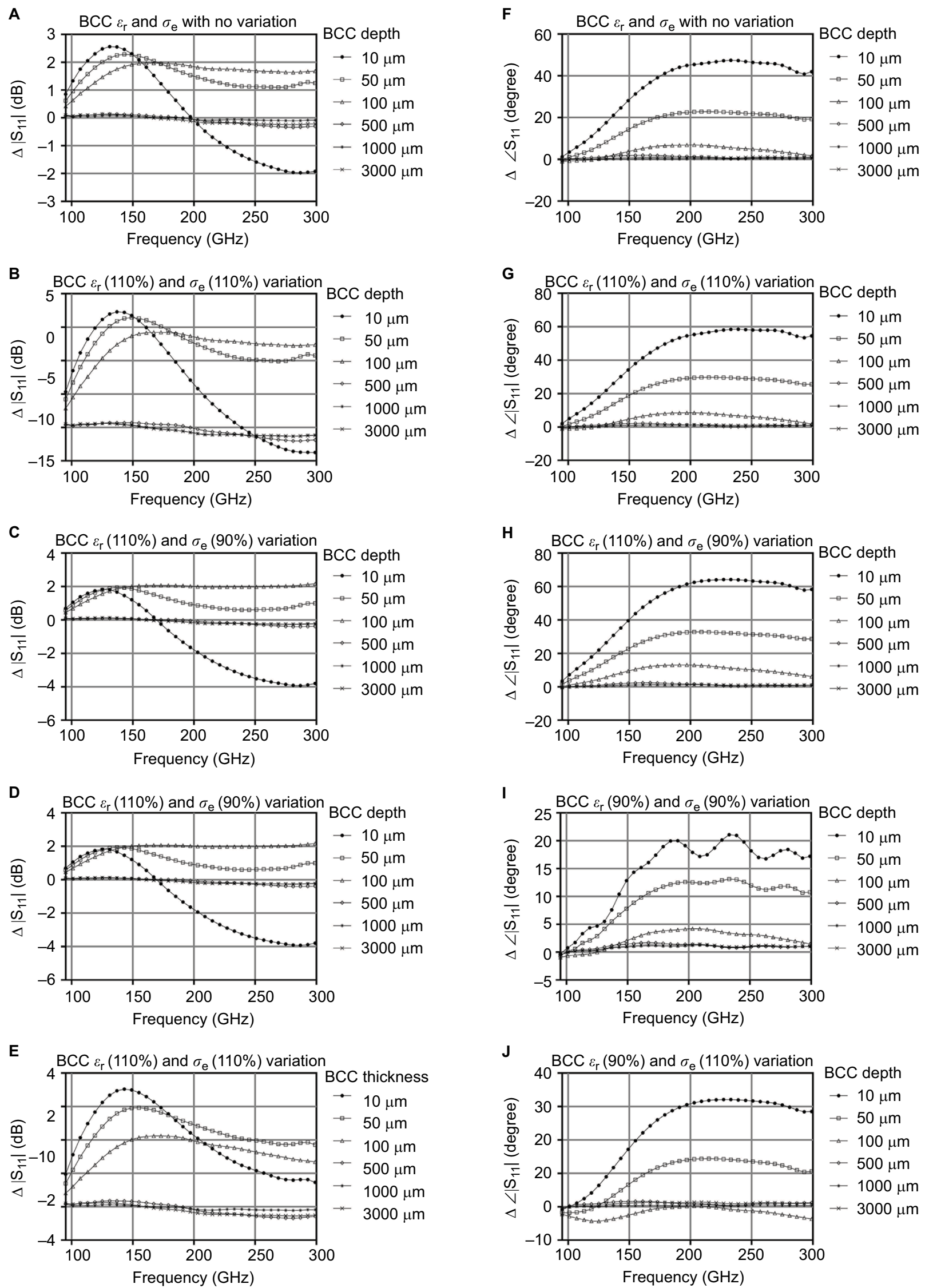

Figure 6 Simulation results of scenario 2 (BCC layer embedded in healthy skin at depths of 10, 50, 100, 500, 1000, and 3000 $\mu$ m): (A-E) shows differences in magnitude $(\Delta|\mathrm{SI} I|)$ and $(\mathbf{F}-\mathrm{J})$ shows phase $(\Delta \angle \mathrm{SI} \mathrm{I})$ of the reflection coefficient, with $\pm 10 \%$ variation of BCC's $\varepsilon_{\mathrm{r}}$ and $\sigma_{\mathrm{e}}$.

Abbreviation: $\mathrm{BCC}$, basal cell carcinoma. 
offers useful information only when BCC is referenced to the adjacent normal skin. Future studies including detailed mathematical modeling and classifications are required in order to further determine the thickness, the location, and dielectric properties over a frequency range.

\section{Conclusion}

Studies and simulation results of a mm-wave rectangular glass filled probe using $\mathrm{CW}$ were carried out for the detection of skin conditions. In this study, normal skin and BCC skin EM models, with $\pm 10 \%$ variation in the $\mathrm{BCC}$ complex dielectric properties, were created and simulated with a high-frequency EM simulator ANSYS HFSS. The simulation results validated that the technique provides a satisfactory sensitivity to detect BCC while providing a small footprint and adequate sensing depth. Depending on the frequency range, either amplitude or phase could be the preferred parameter for the detection of maximum contrast between BCC and normal skin. We concluded that a dual-band system in silicon implementation could be ideal for this application. While a VNA was proposed as a transmitter and receiver in conjunction with the probe, the authors hope that the present work can be extended to an advanced fabricated VNA probe integrated into a single small device in the future, providing a preamble to a compact handheld portable device that can be easily deployed and used. It should be noted that although this concept was validated in simulations, a final decisive conclusion of the usefulness of this concept should only be made after performing in vivo experiments. Hence the next step to validate this concept is to perform a preclinical study using animal models ${ }^{51}$ consisting BCC and normal control skins followed by in vitro study with human skin samples. A statistical study should also be performed to validate its sensitivity and specificity.

\section{Disclosure}

The authors report no conflicts of interest in this work.

\section{References}

1. AIHW, AACR. Cancer in Australia: An Overview, 2006. 2007. Contract No.: CAN 32.

2. AIHW. Cancer in Australia: In Brief 2014. 2015. Contract No.: CAN 89.

3. Hirth G. Improving public health relating to ultra-violet radiation exposure - innovations and plans at Arpansa. Science and the Art of Radiation Protection. Wollongong, Australia: 2017.

4. Malvehy J, Puig S. Follow-up of melanocytic skin lesions with digital total-body photography and digital dermoscopy: a two-step method. Clin Dermatol. 2002;20(3):297-304.

5. Menzies SW, Emery J, Staples M, et al. Impact of dermoscopy and short-term sequential digital dermoscopy imaging for the management of pigmented lesions in primary care: a sequential intervention trial. $\mathrm{Br}$ J Dermatol. 2009;161(6):1270-1277.
6. McBain CA, Henry AM, Sykes J, et al. X-ray volumetric imaging in image-guided radiotherapy: the new standard in on-treatment imaging. Int J Radiat Oncol Biol Phys. 2006;64(2):625-634.

7. Kimball AB, Summers RM, Turner M, et al. Magnetic resonance imaging detection of occult skin and subcutaneous abnormalities in juvenile dermatomyositis: implications for diagnosis and therapy. Arthritis Rheum. 2000;43(8):1866-1873.

8. Barata C, Ruela M, Francisco M, Mendonca T, Marques JS. Two systems for the detection of melanomas in dermoscopy images using texture and color features. IEEE Sys J. 2014;8(3):965-979.

9. Mendi E, Yogurtcular C, Sezgin Y, Bayrak C. Automatic mobile segmentation of dermoscopy images using density based and fuzzy c-means clustering. IEEE Int Symp Med Meas Appl. 2014:1-6.

10. Do TT, Zhou Y, Zheng H, Cheung NM, Koh D. Early melanoma diagnosis with mobile imaging. Conf Proc IEEE Eng Med Biol Soc. 2014;2014:6752-6757. doi: 10.1109/EMBC.2014.6945178.

11. Abuzaghleh O, Barkana BD, Faezipour M. Automated skin lesion analysis based on color and shape geometry feature set for melanoma early detection and prevention. IEEE Long Island Systems, Applications and Technology Conference (LISAT). Farmingdale, NY, USA: 2014.

12. Wang Y, Xu D, Yang S, Xing D. Toward in vivo biopsy of melanoma based on photoacoustic and ultrasound dual imaging with an integrated detector. Biomed Opt Express. 2016;7(2):279-286.

13. Godoy SE, Ramirez DA, Myers SA, et al. Dynamic infrared imaging for skin cancer screening. Infrared Phys Technol. 2015;70:147-152.

14. Madani I, Duthoy W, Derie C, et al. Positron emission tomographyguided, focal-dose escalation using intensity-modulated radiotherapy for head and neck cancer. Int J Radiat Oncol Biol Phys. 2007;68(1):126-135.

15. Berry E, Walker GC, Fitzgerald AJ, et al. Do in vivo terahertz imaging systems comply with safety guidelines? J Laser Appl. 2003;15(3):192-198.

16. Zeni O, Gallerano GP, Perrotta A, et al. Cytogenetic observations in human peripheral blood leukocytes following in vitro exposure to $\mathrm{THz}$ radiation: a pilot study. Health Phys. 2007;92(4):349-357.

17. Jansen ED, Wilmink GJ, Thomas RJ, et al. Quantitative investigation of the bioeffects associated with terahertz radiation. SPIE BiOS. 2010;7562: $75620 \mathrm{~L}$

18. Lane JA, Saxton JA. Dielectric dispersion in pure polar liquids at very high radio-frequencies. I. Measurements on water, methyl and ethyl alcohols. Proc Royal Soc A Math Phys Eng Sci. 1952;213(1114):400-408.

19. Federici JF, Schulkin B, Huang F, et al. THz imaging and sensing for security applications - explosives, weapons and drugs. Semicond Sci Technol. 2005;20(7):S266-S280.

20. McAuley I, Young L, Gradziel M, et al. Millimetre-wave and terahertz imaging systems with medical applications. Conference Digest of the 2006 Joint 31st International Conference on Infrared and Millimeter Waves and 14th International Conference on Terahertz Electronics. 2006:371.

21. Mittleman DM, Gupta M, Neelamani R, et al. Recent advances in terahertz imaging. Appl Phys B Lasers Opt. 1999;68(6):1085-1094.

22. Topfer F, Emtestam L, Oberhammer J. Dermatological verification of micromachined millimeter-wave skin-cancer probe. IEEE MTT-S Int Microw Symposium (IMS). 2014.

23. Hadjiloucas S, Karatzas LS, Bowen JW. Measurements of leaf water content using terahertz radiation. IEEE Trans Microw Theory Tech. 1999;47(2):142-149.

24. Ross KFA, Gordon RE. Water in malignant tissue, measured by cell refractometry and nuclear magnetic resonance. $J$ Microsc. 1982;128(1):7-21.

25. Suntzeff V, Carruthers C. The water content in the epidermis of mice undergoing carcinogenesis by methylcholanthrene. Cancer Res. 1946;6:574-577.

26. Chen JH, Avram HE, Crooks LE, Arakawa M, Kaufman L, Brito AC. In vivo relaxation times and hydrogen density at $0.063-4.85 \mathrm{~T}$ in rats with implanted mammary adenocarcinomas. Radiology. 1992;184(2):427-434. 
27. Schepps JL, Foster KR. The UHF and microwave dielectric properties of normal and tumour tissues: variation in dielectric properties with tissue water content. Phys Med Biol. 1980;25(6):1149-1159.

28. Ronne C, Thrane L, Astrand PO, et al. Investigation of the temperature dependence of dielectric relaxation in liquid water by $\mathrm{THz}$ reflection spectroscopy and molecular dynamics simulation. J Chem Phys. 1997;107(14):5319-5331.

29. Sasaki K, Wake K, Watanabe S. Measurement of the dielectric properties of the epidermis and dermis at frequencies from $0.5 \mathrm{GHz}$ to $110 \mathrm{GHz}$. Phys Med Biol. 2014;59(16):4739-4747.

30. Siebert KJ, Quast H, Leonhardt R, et al. Continuous-wave all-optoelectronic terahertz imaging. Appl Phys Lett. 2002;80(16):3003-3005.

31. Woodward RM, Wallace VP, Arnone DD, Linfield EH, Pepper M. Terahertz pulsed imaging of skin cancer in the time and frequency domain. J Biol Phys. 2003;29(2-3):257-259.

32. Wallace VP, Fitzgerald AJ, Pickwell E, et al. Terahertz pulsed spectroscopy of human basal cell carcinoma. Appl Spectrosc. 2006;60(10):1127-1133.

33. TeraView. Study to Distinguish Between Healthy Tissue and Basal Cell Carcinoma. 2017.

34. Kim JM, Oh D, Yoon J, et al. In vitro and in vivo measurement for biological applications using micromachined probe. IEEE Trans Microw Theory Tech. 2005;53(11):3415-3421.

35. Kim JM, Cho S, Kim N, et al. Planar type micromachined probe with low uncertainty at low frequencies. Sensor Actuat A Phys. 2007;139(1-2):111-117.

36. Byoungjoong K, Park JH, Cho J, et al. Novel low-cost planar probes with broadside apertures for nondestructive dielectric measurement of biological materials at microwave frequencies. IEEE Trans Microw Theory Tech. 2005;53(1):134-143.

37. Danylyuk S, Poppe U, Kadlec F, et al. Broadband microwave-to-terahertz near-field imaging. IEEE/MTT-S International Microwave Symposium, 2007. Honolulu, HI, USA: 2007.

38. Hasch J, Topak E, Schnabel R, et al. Millimeter-wave technology for automotive radar sensors in the $77 \mathrm{GHz}$ frequency band. IEEE Trans Microw Theory Tech. 2012;60(3):845-860.

39. Infineon [webpage on the Internet]. Available from: https://www.infineon.com. Accessed June 26, 2017.
40. SiBEAM. SiBEAM Introduces USB 3.0 802.11ad Reference Design Delivering Wireless Connectivity at Multi-Gigabit Speeds. Available from: http://www.sibeam.com/en/News/2016/20160104SiBEAMUS B3080211ad.aspx. Accessed June 26, 2017.

41. Shuster SAM, Black MM, McVitie EVA. The influence of age and sex on skin thickness, skin collagen and density. $\mathrm{Br} J$ Dermatol. 1975;93(6):639-643.

42. Laurent A, Mistretta F, Bottigioli D, et al. Echographic measurement of skin thickness in adults by high frequency ultrasound to assess the appropriate microneedle length for intradermal delivery of vaccines. Vaccine. 2007;25(34):6423-6430.

43. Cigna E, Tarallo M, Maruccia M, Sorvillo V, Pollastrini A, Scuderi N. Basal cell carcinoma: 10 years of experience. J Skin Cancer. 2011;2011:476362.

44. Pickwell E, Fitzgerald AJ, Cole BE, et al. Simulating the response of terahertz radiation to basal cell carcinoma using ex vivo spectroscopy measurements. J Biomed Opt. 2005;10(6):064021.

45. Meaney PM, Gregory AP, Seppala J, Lahtinen T. Open-ended coaxial dielectric probe effective penetration depth determination. IEEE Trans Microw Theory Tech. 2016;64(3):915-923.

46. Pozar DM. Microwave Engineering. 3 ed. New York: Wiley; 2004.

47. Hasgall PA, Gennaro FD, Baumgartner C [webpage on the Internet], et al. IT'IS Database for Thermal and Electromagnetic Parameters of Biological Tissues 2015. 2018. Available from: www.itis.ethz.ch/ database. Accessed June 26, 2018.

48. Sandby-Moller J, Poulsen T, Wulf HC. Epidermal thickness at different body sites: relationship to age, gender, pigmentation, blood content, skin type and smoking habits. Acta Derm Venereol. 2003;83(6):410-413.

49. Shopov S, Balteanu A, Hasch J, et al. A 234-261-GHz 55-nm SiGe BiCMOS Signal Source with 5.4-7.2 dBm Output Power, 1.3\% DCto-RF efficiency, and 1-GHz Divided-Down Output. IEEE J Solid State Circuits. 2016;51(9):2054-2065.

50. Truong BC, Tuan HD, Fitzgerald AJ, Wallace VP, Nguyen HT. High correlation of double Debye model parameters in skin cancer detection. Conf Proc IEEE Eng Med Biol Soc. 2014;2014:718-721.

51. Chaudhary SC, Waseem M, Rana M, et al. Naproxen inhibits UVBinduced basal cell and squamous cell carcinoma development in Ptch1( $( \pm)$ SKH-1 hairless mice. Photochem Photobiol. 2017;93(4):1016-1024.
Medical Devices: Evidence and Research

\section{Publish your work in this journal}

Medical Devices: Evidence and Research is an international, peerreviewed, open access journal that focuses on the evidence, technology, research, and expert opinion supporting the use and application of medical devices in the diagnosis, monitoring, treatment and management of clinical conditions and physiological processes. The identification of novel

\section{Dovepress}

devices and optimal use of existing devices which will lead to improved clinical outcomes and more effective patient management and safety is a key feature. The manuscript management system is completely online and includes a quick and fair peer-review system. Visit http://www. dovepress.com/testimonials.php to read real quotes from authors. 